\title{
Limits of the Efficiency of Imaging with Obstructing Apertures
}

\author{
A.T. Mohammed \\ Department of Astronomy, College of Science, University of Baghdad, Jadiria, \\ Iraq, Email: arobie33@yahoo.com. \\ حدو الهاءة عند التصوبر بلستخدلم فتحلت معرقلة للضوء

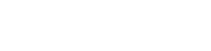

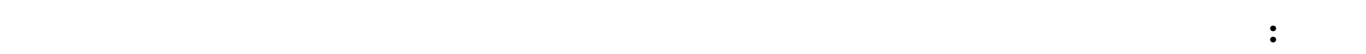

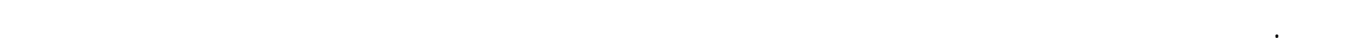

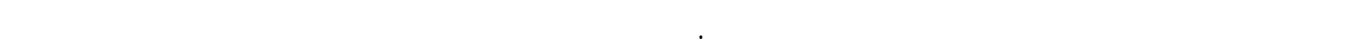 \\ لمستخدلم نصف قطر الإعلةة المركزية لكبر او يساوي ققريباً 0.6مرة من نصف قطر الفتحة الاصلية.
}

\begin{abstract}
Two-dimensional numerical solutions are carried out to asses the quality of obstructing apertures in terms of the diffraction limited resolution. This include the quality of the point spread function (psf), the modulation transfer function $(M T F)$, and an image of double lines. These are average intensity of the $p s f(A I)$, maximum intensity of the $p s f,(M I)$, full width at half maximum of the $p s f(F W)$ average frequency components of $M T F(A F C)$, and average side loops of an image of a double lines. The results indicate that the separation of the two lines becomes recognizable using central obstruction of radius equal to or greater than approximately 0.6 times the radius of the primary aperture.
\end{abstract}

KEYWORDS: Obstructing apertures, optical systems, resolution, fourier transform, image quality.

\section{Introduction}

A

11 telescopes have an inherent limitation to their angular resolution due to the diffraction of light at the telescope aperture. The diffraction causes an optical system to behave as a low-pass filter in the formation of an image. The cut-off frequency is directly determined by the shape and size of the limiting pupil in the optical system. The incoming light is approximately a plane wave since the source of the light is so far away.

There are several criteria for analyzing the performance of an optical imaging systems. The Rayleigh criterion is generally regarded as a fundamental limit in predicting the performance of optical imaging systems.

According to the Rayleigh criterion, the theoretical resolving power of $5 \mathrm{~m}$ optical telescope $(\lambda=400 \mathrm{~nm})$ are $\cong 0.02 "$. This value shows the relationship between the resolving power and the telescope apertures. In addition to that criterion, measurements of $M T F$, strehl ratio, and diffraction limited resolution are also very well 


\section{A.T. MOHAMMED}

considered in quantifying optical systems (William and Bucklund 1989, Harvey and Ftaclas 1995, Brummelaar and Bagnuolo 1995, Brammelaar et al. 1994, Baldwin et al. 2001, Granieri et al. 1998, Jean-Marc et al. 2004, Mohammed et al. 1990, Milanfar and Shakouri, 2002).

Many studies have been presented in the literatures concerning imaging with obstructing aperture (Fienup 2000, Mohammed 2006, Chakraborty and Thompson 2005).

The aim of this paper is to present the quantitative assessment of the limitations imposed by obstructing apertures on the psf and MTF in order to determine the constraints on the efficiency of imaging with such apertures.

\section{Theory}

The fundamental equation to be used for the formation of an image by an ideal optical system is given by:

$$
\begin{gathered}
i(x, y)=\iint_{-\infty}^{\infty} o\left(x^{\prime}, y^{\prime}\right) p s f\left(x-x^{\prime}, y-y^{\prime}\right) d x^{\prime} d y^{\prime} \\
i(x, y)=o(x, y) \otimes p s f(x, y)
\end{gathered}
$$

Equations (1) and (2) are equivalent and representing a convolution equation. Where $i(x, y)$ is the observed image intensity, $o(x, y)$ is the object intensity, $p s f(\mathrm{x}, \mathrm{y})$ represents the image blurring function caused by the imaging system and $\otimes$ denotes convolution operator.

The Fourier transform of (2) is given by:

$$
\mathrm{I}(\mathrm{u}, \mathrm{v})=\mathrm{O}(\mathrm{u}, \mathrm{v}) \cdot \mathrm{T}(\mathrm{u}, \mathrm{v})
$$

where $I(u, v)$ and $O(u, v)$ are, respectively, the complex Fourier transforms of the image intensity $i(x, y)$, and the object intensity $o(x, y) ; T(u, v)$ which represents the Fourier transform of the $p s f$, is an important function known as the optical transfer function $(O T F)$. The modulation or amplitude of the complex function $T(u, v)$ is called $M T F$. In general, the resolution of an imaging system is limited only by the luck of large optical elements that are free from inherent distortions.

Now consider an extremely distant quasimonochromatic point source located on the optical axis of a simple imaging system. In the absence of atmospheric turbulence, this source would generate a plane wave normally incident on the lens. In the presence of the atmosphere, the plane wave incident on the inhomogeneous medium propagates into the medium, and ultimately a perturbed wave falls on the lens. The field distribution incident on the lens can be expressed as,

$$
U(\eta, \gamma)=e^{i \phi(\eta, \gamma)}
$$

where $\phi(\eta, \gamma)$ is the random phase of the incident wavefront and the variables $(\eta, \gamma)$ represent distances in the pupil function. The instantaneous $p s f$ of the entire telescope atmosphere system is given by:

$$
p s f(x, y)=|F T[H(\eta, \gamma) U(\eta, \gamma)]|^{2}
$$




\section{LIMITS OF THE EFFICIENCY OF IMAGING WITH OBSTRUCTING APERTURES}

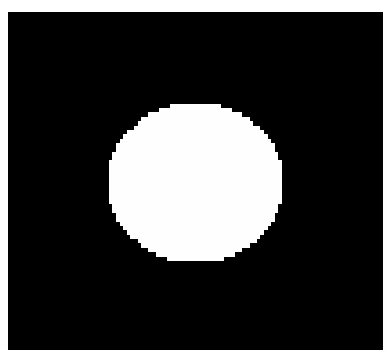

(a)

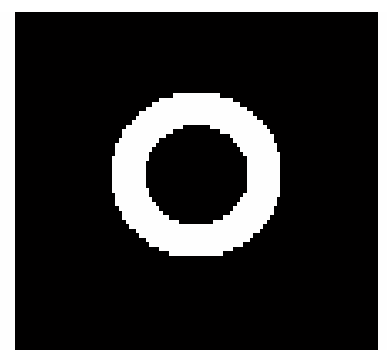

(b)

Figure 1. Telescope apertures:(a) Uniform ( $\varepsilon=0$ ), (b) $\varepsilon=0.6$

where $H_{(\eta, \gamma)}$ represents the pupil function and $F T$ denotes Fourier transform operator. The corresponding OTF is the Fourier transform of the psf, thus

$$
\mathrm{T}(\mathrm{u}, \mathrm{v})=\mathrm{FT}[\mathrm{psf}(\mathrm{x}, \mathrm{y})]
$$

Equation (6) can also be written in terms of the pupil function and the field distribution incident on the lens as,

$$
\begin{array}{r}
T(u, v)=\iint_{-\infty}^{\infty} H(\eta, \gamma) H^{*}\left(\eta-\eta^{\prime}, \gamma-\gamma^{\prime}\right) . \\
\\
U(\eta, \gamma) U^{*}\left(\eta-\eta^{\prime}, \gamma-\gamma^{\prime}\right) d \eta^{\prime} d \gamma^{\prime}
\end{array}
$$

where * denotes complex conjugate. The variables $\eta$ and $\gamma$ are related to the Fourier space variables $u$ and $v$ by $\eta=\lambda f u, \quad \gamma=\lambda f v$, where $\lambda$ is the wavelength and $f$ is the focal length.

\section{Simulations}

The size of the pupil function $H(u, y)$ is taken to be a two dimensional circular function of radius $R$ and of unity magnitude; this array $(M \times N)$ is of size $512 \times 512$ pixels. This size is taken as large as possible in order to keep the theoretical diffraction limiting resolution vanishing to zero inside this array. This aperture is said to be a uniform aperture.

The central obstructing aperture is simulated by calculating the parameter $\varepsilon$. This parameter represents the ratio of the radius of obstructing circle ( $r$ ) to the radius of the uniform aperture, $R$, i.e., $\varepsilon=r / R$.

Telescope apertures of $\varepsilon=0$ and $\varepsilon=0.6$ are demonstrated in Figure 1.

We consider the object to be imaged is an extremely distant quasimonochromatic point source located on the axis of an optical telescope. In the absence of atmospheric turbulence, this source would generates a plane wave $[\phi(\eta, \gamma)=0$ and $U(\eta, \gamma)=1]$.

The $p s f s$ are computed via Equation (5). The perspective plots of the central regions of these $p s f s$ (14 pixels by 14 pixels ) are shown in Figure 2. 


\section{A.T. MOHAMMED}

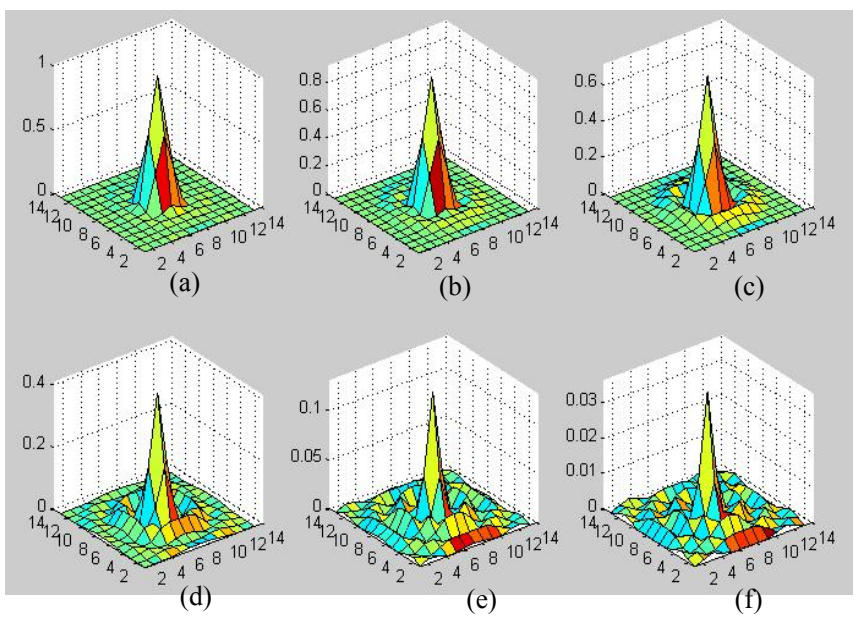

Figure 2. Perspective plots of the central regions of psf's, (a) Uniform aperture,(b) $\varepsilon=0.2$,(c) $\varepsilon=0.4$, (d) $\varepsilon=0.6$ and (e) $\varepsilon=0.8$ (f) $\varepsilon=0.9$.

It should be pointed out here that the central spikes of the $p s f s$ are very sharp. This is because $R$ is taken to be very large, $R=120$ pixels.

The line plots through the centre of these regions are shown in Figure 3.

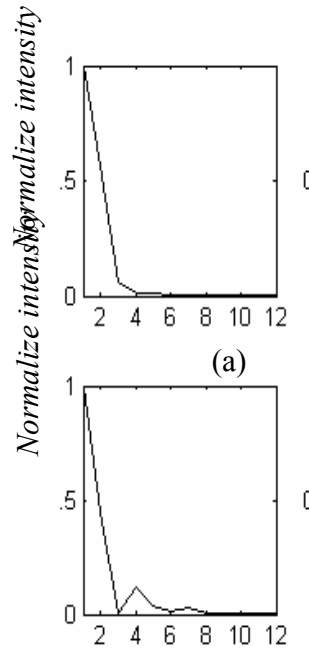

(d)

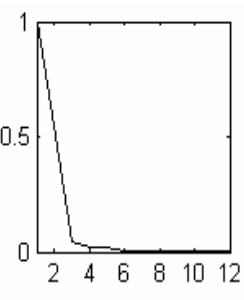

(b)

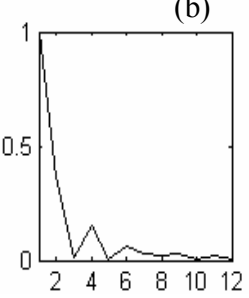

(e)
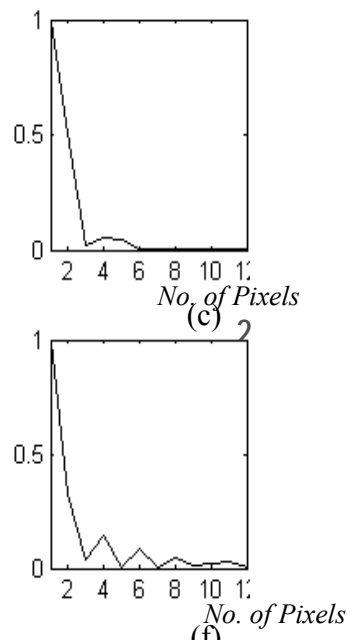

Figure 3. Plots through the central regions of the psfs. (a) Uniform aperture,(b) $\varepsilon=0.2$,(c) $\varepsilon=0.4$,(d) $\varepsilon=0.6$,(e) $\varepsilon=0.8$ and (f) $\varepsilon=0.9$.

The MTFs are also computed according to Equation (6) or (7) respectively and absolute values are taken for $T(u, v)$. The results are shown in Figure 4. 


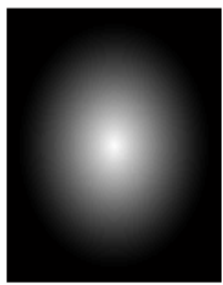

(a)

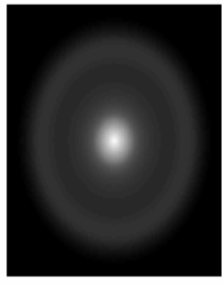

(d)

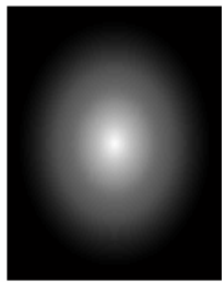

(b)

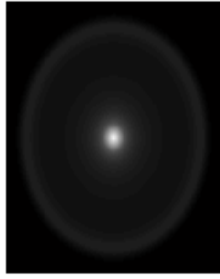

(e)

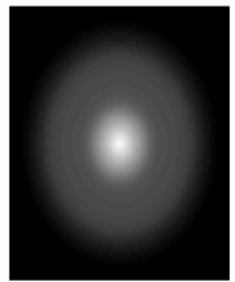

(c)

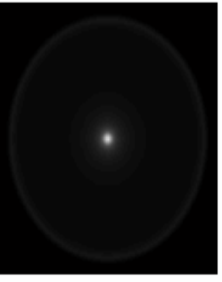

(f)

Figure 4. MTF's (a) Uniform aperture,(b) $\varepsilon=0.2$,(c) $\varepsilon=0.4$,(d) $\varepsilon=0.6$ (e) $\varepsilon=0.8$ and (f) $\varepsilon=0.9$

The above images are then normalized to their maximum values according to the following equation:

$$
\operatorname{MTF}_{N}(x, y)=\frac{\operatorname{MTF}(x, y)}{\operatorname{MTF}(0,0)}
$$

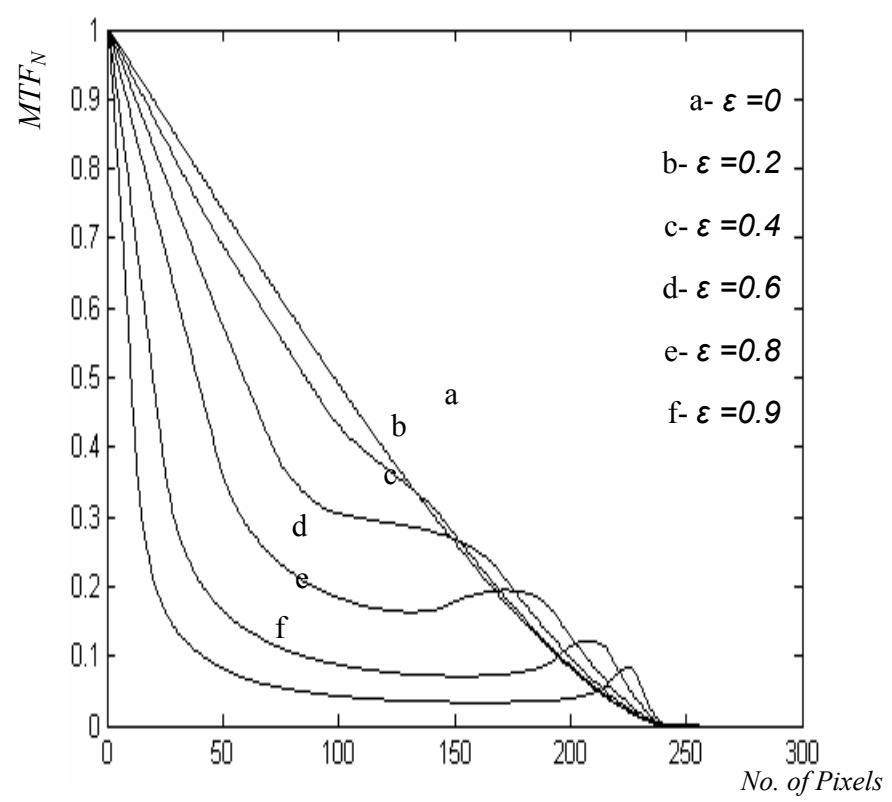

Figure 5. Central plots through Figure 4.

where $\operatorname{MTF}(0,0)$ represents the value of the central spike.

The central lines of the normalized $M T F S$ (i.e. $M T F_{N}$ ) are plotted in Figure 5. 


\section{A.T. MOHAMMED}

The $p s f s$ at different values of $\varepsilon$ are convolved with the double line object presented in Figure 6(a). The lines are one pixel wide separated by a distance of 2 pixels. This value is chosen because the full width of the $p s f$ of the uniform aperture is 3 pixels. The results are shown in Figure 6.

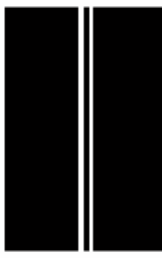

(a)

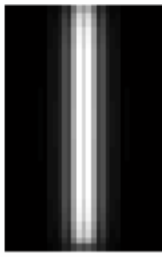

(e)

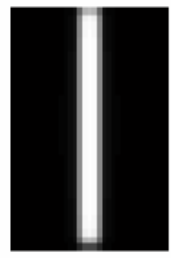

(b)

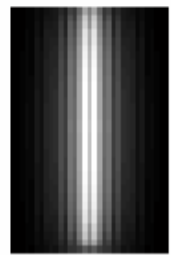

(f)

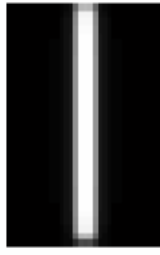

(c)

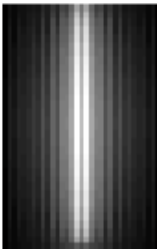

$(\mathrm{g})$

Figure 6. Line Convolutions:(a) Uniform aperture, (b) $\varepsilon=0.2$, (c) $\varepsilon=0.4$, (d) $\varepsilon=0.6$, (e) $\varepsilon=0.8$ and (f) $\varepsilon=0.9$.

The images in Figure 6 are then normalized to one at their maximum values and the central lines through these images are plotted. The results are presented in Figure 7.

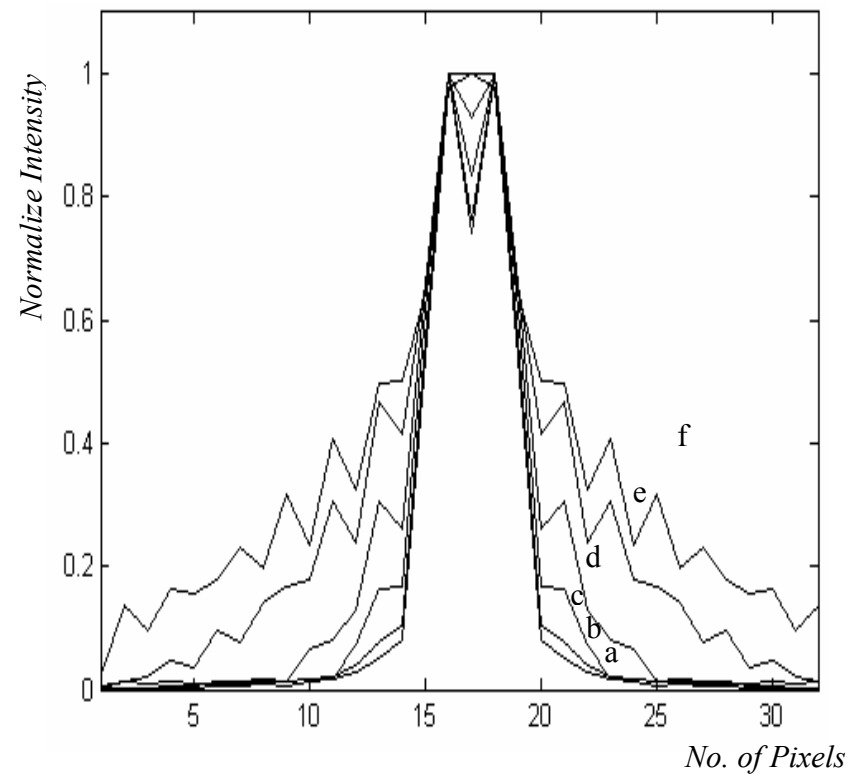

Figure 7. Normalized Intensities through the center of the images in Figure 6. (a) Uniform aperture,(b) $\varepsilon=0.2$,(c) $\varepsilon=0.4,(\mathrm{~d}) \varepsilon=0.6,(\mathrm{e}) \varepsilon=0.8$ and (f) $\varepsilon=0.9$. 


\section{LIMITS OF THE EFFICIENCY OF IMAGING WITH OBSTRUCTING APERTURES}

For $\varepsilon=0$, the line plot shows a little peak at the center. As $\varepsilon$ increases, the separation of the double lines becomes recognizable and the side loops become severe.

The normalized maximum intensity values $(M I)$ of the $p s f s$ are calculated and presented in the Table 1 given below as follows. First, the maximum value of the $p s f$ of the uniform aperture $(\varepsilon=0)$ is calculated. Secondly, the maximum intensity values at different $\varepsilon$ are divided by the maximum value at $\varepsilon=0$.

This measure is taken in order to examine the dropness in the value of the central spike of the psf as a function of $\varepsilon$.

Table 1. The variation of MI, AI, FW, AFC and SL at different values of $\varepsilon$.

\begin{tabular}{|c|c|c|c|c|c|}
\hline$\varepsilon$ & $M I$ & $A I$ & $F W$ & $A F C$ & $S L$ \\
\hline 0 & 1 & 1 & 3 & 1 & 1 \\
\hline 0.2 & 0.9224 & 1.0412 & 3 & 0.9604 & 1.0808 \\
\hline 0.4 & 0.707 & 1.19 & 3 & 0.8406 & 1.2373 \\
\hline 0.6 & 0.411 & 1.56 & 1 & 0.6410 & 1.5328 \\
\hline 0.8 & 0.13 & 2.77 & 1 & 0.3607 & 2.3403 \\
\hline 0.9 & 0.0362 & 5.26 & 1 & 0.1902 & 3.0122 \\
\hline
\end{tabular}

The average intensity values $(A I)$ of the $p s f$ at different $\varepsilon$ is computed by:

$$
\mathrm{A} I=\sum_{\mathrm{y}=1 \mathrm{x}=1}^{\mathrm{M}} \sum^{\mathrm{N}} p s f(x, y) / \sum_{y=1}^{M} \sum_{x=1}^{N} p s f_{U}(\mathrm{x}, \mathrm{y})
$$

where $p s f_{U}(x, y)$ is the $p s f$ at $\varepsilon=0$.

The average frequency components $(A F C)$ of $\operatorname{MTF}(u, v)$ at different $\varepsilon$ are computed by:

$$
\mathrm{AFC}=\sum_{u=1}^{\mathrm{M}} \sum_{v=1}^{\mathrm{N}} \mathrm{MTF}(u, v) / \sum_{u=1}^{M} \sum_{v=1}^{N} M T F_{U}(u, v)
$$

where $\operatorname{MTF}_{U}(u, v)$ is the $M T F$ at $\varepsilon=0$.

The full width of the $p s f(F W)$ at different $\varepsilon$ are also calculated and presented in this table. These values indicate the width of the central spike of the $p s f s$.

The summation of all values that located outside the double lines of the $p s f s$ at different $\varepsilon$ (see Figure.6) are computed and the values are then divided by the corresponding value of the uniform aperture. The results are describing the actual weight of the side loops $(S L)$.

\section{Conclusions}

The following conclusions could be drawn :

1. The $M I$ value decreases by a factor of $\cong 30$ as $\varepsilon$ goes from $\varepsilon=0$ to $\varepsilon=0.9$. This great reduction in the height of central spike will enhance the probability of detecting faint companion (Figure 7 describes normalized intensity not actual intensity).

2. As $\varepsilon$ increases, $A I$ increases and at $\varepsilon=0.9, A I$ increases by a factor of $\cong 5$. This is of course will create artifacts in the observed images (see Figure. 2).

3. $F W$ reduced dramatically as $\varepsilon$ increases. For $\varepsilon=0.6, F W$ reduces by a factor of 3 . This will enhance the resolution by a factor of 3 . 


\section{A.T. MOHAMMED}

4. $A F C$ reduces by nearly a factor of $\cong 5$ at $\varepsilon=0.9$. This is because the build up of the side loops in the psfs. $A F C$ is inversely proportional to $\varepsilon$.

5. The $S L$ increases by a factor of 3 at $\varepsilon=0.9$. SL is linearly proportional to $\varepsilon$.

\section{References}

BALDWIN, J.E., TUBBS, R.N., COX, G.C., MACKAY, C.D., WILSON, R.W. and ANDERSON, M.I. 2001. Diffraction limited $800 \mathrm{~nm}$ imaging with $2.56 \mathrm{~m}$ Nordic optical telescope. Astronomy and Astrophysics 368: L1-L4.

BRUMMELAAR, T.A. and BAGNUOLO, W.G. 1995. Strehl ratio and visibility in long baseline stellar interferometry. Optics Letters 20: 521-23.

BRUMMELAAR, T.A., BAGNUOLO, W.G. and RIDGWAY, S.T. 1994. Strehl ratio and coherence loss in long baseline interferometry. Technical Report, Center for High Angular Resolution Astronomy Georgia State University, No. 6.

CHAKRABORTY, A. and THOMPSON, L.A. $2005 \cdot 10^{-7}$ contrast ratio at $4.5^{\lambda} / \mathrm{D}$ :New results obtained in laboratory experiments using nonfabricated coronograph and multi-Gaussian shaped pupil masks. Optics express 13: 2394.

DEBES, J. 2003. High contrast imaging with Gaussian aperture pupil mask. astro-ph 0301051, V1.

FIENUP, J.R. 2000. MTF and integration time versus fill factor for sparse-aperture imaging system. Proc. SPIE. 4091 A-06 San Diego, CA.

GRANIERI, S., ENRIQUE, E. and FURLAN, W.D. 1998. Performance analysis of optical imaging systems based on fractional Fourier transform. Journal of Modern Optics 45: 1797-07.

HARVEY, J.E and FTACLAS, C. 1995. Diffraction effects of telescope secondary mirror spiders on various image quality criteria. Applied Optics 34: 6337-49.

JEAN-MARC, D., LEGER, D., ROGUES, S. and VALORGE, C. 2004. Modulation transfer function estimation from non specific images. Optical Engineering 43: 1355-65 DONAL, F.O'shea; Ed.

MILANFAR, P. and SHAKOURI, A. 2002. Statistical analysis of diffraction limited images. IEEE ICIP.

MOHAMMED, A.T., RASHID, N.M. and SADIK, A.R. 1990. Computer simulations of astronomical objects as seen by ground based optical telescope. Optics and Lasers in Engineering 12: 223-43.

MOHAMMED, A.T. 2006. Quantitative analysis of imaging with apodized square apertures. Iraqi Journal of Science 47: 255-61.

SONG, JONG.SUP., LEE YUN WOO, SONG JAE BONG, LEE IN WON, YANG HO-SOON, CHOI YOUNGWAN, and DUAL JAE HEUNGO JO. 2002. Dual testing of a large aperture optical system. Proc. SPIE, Interferometry XI:Applications, 4778: 227-36.

WILLIAM, C. and BECKLUND, O. 1989. Introduction to the Optical Transfer Function. Wiley Series in Pure and Applied Optics.

Received 7 January 2007

Accepted 23 October 2007 